\title{
Study of Correlation between Vitamin ' $D$ ' Deficiency and Anti-Thyroperoxidase Antibodies in Autoimmune Thyroid Diseases
}

\author{
${ }^{1}$ Preeti Patni, ${ }^{2}$ Tanu Choudhary, ${ }^{3}$ Tarun Sekhri, ${ }^{4}$ Ratnesh Kanwar, ${ }^{5}$ Neeta Uniyal
}

\section{ABSTRACT}

Aim: The aim of this study is to explore the relationship between vitamin D levels and antithyroid peroxidase antibodies (ATPO $\mathrm{Ab})$ in recently diagnosed patients of autoimmune thyroid disease (AITD).

Material and methods: A total of 138 and 30 recently diagnosed female patients of Hashimoto's thyroiditis (HT) and graves' disease (GD) along with 46 healthy females as controls were enrolled in groups I, II and III respectively. In this study 25OHD, PTH, calcium, and thyroid autoantibody [anti-thyroid peroxidase (anti-TPO)] levels were evaluated for all subjects $(n=214)$.

Results: Lower serum 250HD levels were present in groups I and II patients $(12.6 \pm 9.04 \mathrm{ng} / \mathrm{mL}, 10.6 \pm 5.4 \mathrm{ng} / \mathrm{mL}$ respectively) than in group III subjects $(22.8 \pm 6.4 \mathrm{ng} / \mathrm{m}\} \mathrm{L})$, ( $<0.00001)$. Although serum $250 H D$ levels inversely correlated with anti-TPO $(r=-0.32, p=0.0001)$ and parathormone $(\mathrm{PTH})$ $(r=-0.27, p=0.0049)$ but no significant correlation of serum $25 \mathrm{OHD}$ levels was found with TSH $(r=0.04, p=0.07)$ and FT4 levels $(r=0.02, p=0.6)$. Hypothyroid patients (Group I) had higher anti-TPO levels than patient with GD and controls (groups II and III) (p<0.0001).

Conclusion: Our study demonstrated that lower vitamin D levels are found in AITD patients in at present. Severity and prevalence of vitamin D deficiency are higher in GD patients than hypothyroid patients. An inverse correlation was found in this study between antithyroid peroxidase antibodies (ATPO $A b)$ and vitamin $D$ levels. We may propose that vitamin $D$ deficiency is one of the potential factors in the pathogenesis of autoimmune thyroid disorders.

Clinical significance: More larger studies, to evaluate the role of Vitamin D in pathogenesis of AITD and beneficial effect of its supplementation in treatment, are needed.

Keywords: Antithyroid peroxidase, Autoimmune thyroid disease (AITD), Vitamin D.

${ }^{1} \mathrm{HOD},{ }^{2}$ Student, ${ }^{3}$ Scientist and HOD, ${ }^{4}$ Scientist, ${ }^{5}$ Technician

${ }^{1,5}$ Department of Lab Medicine, Institute of Nuclear Medicine and Allied Sciences, New Delhi, India

${ }^{2}$ Army College of Medical Sciences, Delhi, India

${ }^{3,4}$ Endo and Thyro Research, Institute of Nuclear Medicine and Allied Sciences, Army College of Medical Sciences, Delhi

Corresponding Author: Preeti Patni, HOD, Department of Lab Medicine, Institute of Nuclear Medicine and Allied Sciences, New Delhi, India, e-mail: preetipatni1@gmail.com
How to cite this article: Patni $\mathrm{P}$, Choudhary $\mathrm{T}$, Sekhri T, Kanwar R, Uniyal N. Study of Correlation between Vitamin 'D' Deficiency and Anti- Thyroperoxidase Antibodies in Autoimmune Thyroid Diseases. Journal of Medical Academics 2018;1(1):10-14.

\section{Source of support: Nil}

Conflict of interest: None

\section{INTRODUCTION}

Autoimmune thyroid disease (AITD) is a condition in which body interprets the thyroid hormones-T3, T4, and $\mathrm{TSH}$ as threats and starts producing antibodies against the thyroid gland as a protective response. As per the definition of American Thyroid Society-Thyroiditis refers to "inflammation of the thyroid gland" which includes a group of individual disorders causing thyroidal in flammation but presenting in different ways. It may be present with hypothyroidism or hyperthyroidism and with or without goiter. ${ }^{1}$ These AITDs are polygenic diseases which result due to the amalgamation of genetic susceptibilities (Immune-modulating genes and thyroidspecific genes) and environmental factors (irradiation, iodine, drugs, selenium, stress, smoking, infections, etc.), characterized by the production of thyroid-specific autoantibodies and lymphocytic infiltration into the thyroid gland. Three different antibodies are usually produced:

- Thyroid peroxidase antibodies (TPOAb)

- Thyroglobulin antibodies (TgAb)

- Thyroid stimulating hormone receptor antibodies (TRAb)

Various studies showed that most commonly tested antibodies are TPOAbs in hypothyroid patients or when a person is started with drug therapy like lithium or interferon resulting in hypothyroidism.

Autoimmune thyroid diseases (AITD) are, amongst the commonest endocrine disorders worldwide. According to the Indian Journal of Endocrinology and Metabolism- over 42 million people in India are suffering from thyroid diseases. The major epidemiology under this includes hypothyroidism; hyperthyroidism; goiter and iodine deficiency disorders; Hashimoto's thyroiditis; and thyroid cancer. AITD occurs ten times more frequently in women ( $\mathrm{F}: \mathrm{M}=10: 1)$ and is more common in middle age (30 to 50 years). 
Vitamin D is a lipid-soluble vitamin which has an important role in bone and mineral homeostasis. Vitamin D plays an important immunomodulatory role by enhancing the innate immune response while inhibiting the adaptive immune system. ${ }^{2}$ Vitamin D deficiency has become a common problem in the general population. Many recent studies could demonstrate a correlation between autoimmune diseases and vitamin D deficiency, such as rheumatoid arthritis, ${ }^{3}$ systemic lupus erythematous, ${ }^{4}$ systemic sclerosis and autoimmune thyroiditis. It was shown that vitamin D levels were lower in patients with Hashimoto's thyroiditis, an AITD. ${ }^{5}$ Both vitamin $\mathrm{D}$ and thyroid hormone bind to similar receptors called steroid hormone receptors. Vitamin D mediates its effect through binding to vitamin D receptor (VDR), and activation of VDR-responsive genes. While VDR gene polymorphism was found to associate with AITDs. ${ }^{6}$

Because of conflicting results in already published studies, the present study is aimed to examine the correlation between vitamin D deficiency and AITDs, thyroid function, thyroid autoantibodies, and demographic characteristics.

\section{AIMS AND OBJECTIVES}

- The aim of this study is to investigate the relation between vitamin D levels and thyroid antibodies (ATPO Ab) in newly diagnosed patients of AITD.

- The objectives of this study are:

1. To study the demographic characteristics of patients with normal thyroid functions and abnormal thyroid functions (having autoantibodies).

2. To find the vitamin D levels in patients with AITD.

3. To find the vitamin D levels in non-AITD patients.

4. To find the correlation between the vitamin D levels and autoantibodies.

\section{MATERIALS AND METHODS}

The study was approved by Ethics Committees of Army College of Medical Sciences (Project Ref No: IEC/ACMS/ NP-1/2017) and INMAS, DRDO. All the patients and healthy controls were enrolled in the study only after obtaining informed consent.

Study population: A total of 214 (all female) patients were included in this "case-control" type study, living in the NCR region. These subjects were classified into three main categories:

(A). Group I: "Hypothyroid patients"-It included 138 newly diagnosed hypothyroid patients with elevated TPOAb and thyrotrophic hormone (TSH) along with typical hypoechogenicity of the thyroid gland in high-resolution sonography and cytological findings s/o lymphocytic thyroiditis on FNA.
(B). Group II: "Hyperthyroid patients"- It included 30 recently diagnosed hyperthyroid patients with diffused goiter, suppressed TSH levels and elevated free thyroxine (fT4) along with thyroid receptor antibody (TRAb) positivity.

(C). Group III: "Control Group"- Included 46 apparently healthy individuals. Individuals in this group did not have a history of any chronic illness or thyroid diseases which may affect results. They were not on any medication or vitamin D supplements as well.

The inclusion and the exclusion criteria used in the study were as follows:

\section{Inclusion Criteria}

- Willingness for the procedure, i.e., after obtaining informed consent

- Patients who are newly diagnosed cases of thyroid diseases

\section{Exclusion Criteria}

- Patients who are on treatment for hypo or hyperthyroidism

- Patients who are on medication, such as calcium, vitamin $\mathrm{D}$, steroids, antitubercular and antiepileptics, etc., which could affect bone mineral metabolism

- Patients under investigation for any immunological disorder

- In this study all cases were subjected to the followings:

(a) Complete history taking along with clinical examina tion.

(b) Investigations - Blood sample was collected by venipuncture at the fasting state for estimation of serum 25(OH)D, PTH, TSH, $\mathrm{FT}_{4}, \mathrm{FT}_{3}$, and anti-TPO levels. Serum was stored at $-80^{\circ} \mathrm{C}$ for a period of 7 to 10 days until analyzed.

- Vitamin D: Serum 25(OH)D levels, as a mark of Vitamin D status, were estimated by an Electrochemiluminescent immunoassay method (ECL) on Cobas e411, Roche, Germany (Lot No-23900702). Vitamin D deficiency was considered when serum $25(\mathrm{OH}) \mathrm{D}$ levels fell below $20 \mathrm{ng} / \mathrm{mL}$.

- Parathormone: Serum PTH levels were measured by ECL method using Cobas e 411, Roche, Germany (Lot No-22037701), normal range 15 to $68 \mathrm{pg} / \mathrm{mL}$. Serum calcium (Ca) levels were measured with an enzymatic colorimetric assay on Cobas Integra 400, Roche (Normal: 8.6-10.4 mg/dL)

- The thyrotrophic hormone, FT4, FT3and anti-TPO: Serum TSH, FT4, FT3, and anti-TPO levels were also tested by ECL method using Cobas e411, Roche, Germany (Lot no-22637601, 26563101, 25785705, and 17904402). Reference ranges for Serum FT3, FT4, TSH \& ATPO for 


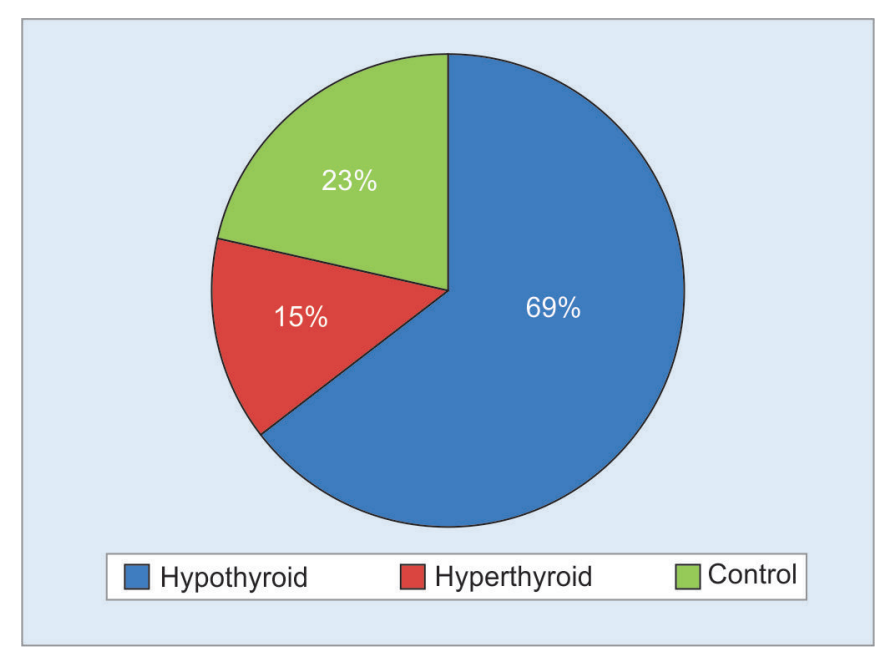

Fig. 1: The participants distribution in different groups

patients are (2.6-6.8 pmol/L), (12-22 pmol/L), (0.27-4.2 $\mu \mathrm{IU} / \mathrm{mL})$ and $(<35 \mathrm{IU} / \mathrm{mL})$ respectively.

\section{Statistical Analyses}

Results were statistically analyzed by SPSS 15 for Windows. The mean and the standard deviation (SD) for all the variables were calculated. Analysis of variance F test (ANOVA) was used to compare the results of all examined cases in all studied groups.

The correlations between serum Vitamin D, Calcium, ATPO and TSH were presented by the correlation coefficient $\left(\mathrm{r}^{2}\right)$. Results considered significant or non-significant when $\mathrm{p}$ value $>$ or $<0.05$, respectively

\section{RESULTS}

Total of 214 cases comprising of 138 patients from group I (Hypothyroid), 30patients from Group II (Hyperthyroidism) and 46 controls were enrolled in this study Figure 1.

Mean age of patients in a study population of Gp I, Gp II and Control Gp was $34.3 \pm 9.5$ yrs, $34.53 \pm 13.69$ yrs, and $24.74 \pm 4.46 y$ rs respectively.

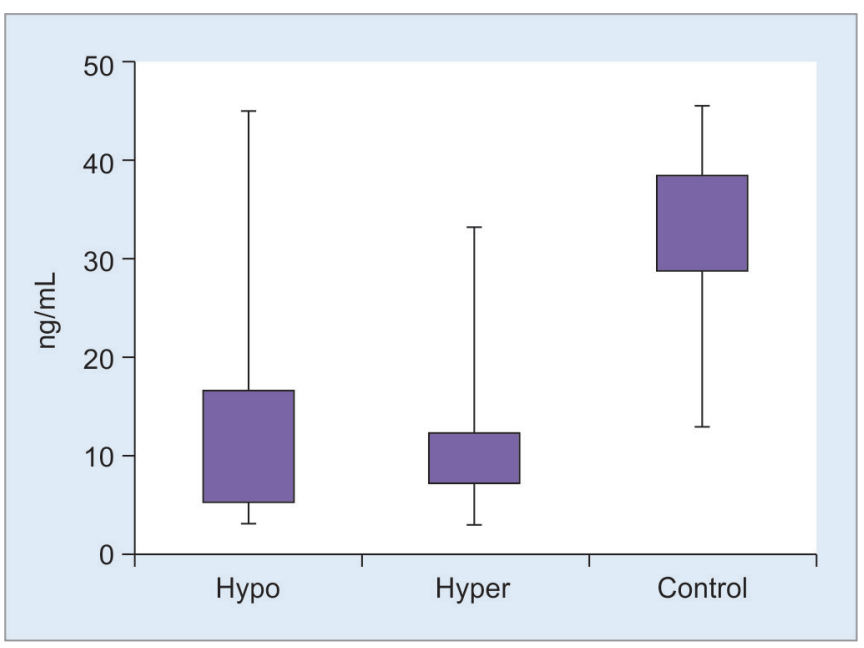

Fig. 2: Vitamin D Levels in each group
Table 1: Mean \pm SD of parameters studied in present study

\begin{tabular}{lllll}
\hline & $\begin{array}{l}\text { Group I } \\
\text { (Hypothyroid) }\end{array}$ & $\begin{array}{c}\text { Group II } \\
\text { (Hyperthyroid) }\end{array}$ & $\begin{array}{l}\text { GroupllI } \\
\text { (Control) }\end{array}$ & \\
\cline { 2 - 4 } Parameter & \multicolumn{3}{c}{ Mean \pm SD } & \multicolumn{1}{c}{$p$-value } \\
\hline TSH & $15.5 \pm 24.2$ & $0.06 \pm 0.06$ & $2.37 \pm 1.03$ & 0.0024 \\
$(\mu \mathrm{lU} / \mathrm{mL})$ & & & & \\
FT3 pmol/L & $4.3 \pm 0.7$ & $9.2 \pm 4.8$ & $4.65 \pm 0.67$ & 0.00001 \\
FT4pmol/L & $14.3 \pm 3.8$ & $26.6 \pm 10.5$ & $16.33 \pm 1.8$ & 0.00001 \\
ATPO $(\mathrm{IU} / \mathrm{mL})$ & $276.8 \pm 168$ & $183.4 \pm 150$ & $6.8 \pm 2.2$ & 0.00001 \\
Vitamin D & $12.6 \pm 9.04$ & $10.6 \pm 5.4$ & $22.8 \pm 6.4$ & 0.00001 \\
PTH $(\mathrm{pg} / \mathrm{mL})$ & $45.7 \pm 25.8$ & $37.44 \pm 12.9$ & $29.2 \pm 21.9$ & 0.0146 \\
Ca2+ $(\mathrm{mg} / \mathrm{dL})$ & $8.9 \pm 0.88$ & $9 \pm 0.35$ & $9.4 \pm 0.3$ & 0.09 \\
\hline
\end{tabular}

The most common presenting complaint in group I and II was a generalized weakness (78\% and 67\% respectively). Other complaints in group I were diffuse swelling $(72 \%)$, pain in throat $(56 \%)$, weight gain $(43 \%)$ and low-grade fever (14\%) while in group II patients reported palpitations $(64 \%)$, weight loss $(58 \%)$ and thyroid swelling (53\%).

The values of vitamin D and ATPO in each group are shown in (Fig 2 and 3) respectively. Table 1 shows Mean \pm S.D values of all studied parameters in each of the studied groups. PTH value did not have a statistical significant difference $(p>0.09)$ between groups.

In this study, hypothyroid patients had significantly higher serum TSH level than that of controls $(p=0.002)$. Serum FT3 and FT4 were significantly high in hyperthyroid patients $(\mathrm{p}=0.00001)$. Group I hypothyroid patients $(n=138)$ had significantly higher average ATPO levels (276.8 IU/mL) as compared to group II Hyperthyroid patients $(183.4 \mathrm{IU} / \mathrm{mL})$. Serum vitamin D levels, they were significantly low in both hypothyroid (group I) and hyperthyroid (group II) than in controls (Fig. 2). The prevalence of vitamin D deficiency was $67 \%$ and $80 \%$ in group I and group II, respectively. Although the levels of vitamin D and PTH levels were lowest in group II (Table. 1), calcium levels were similar between groups.

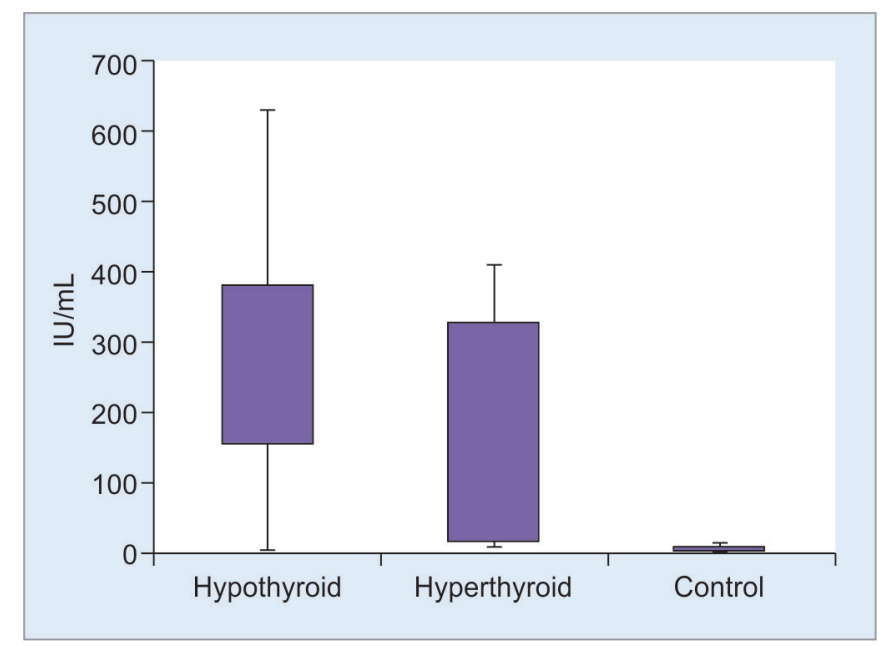

Fig. 3: ATPO levels in each group 
Study of Correlation between Vitamin 'D' Deficiency and Anti-Thyroperoxidase Antibodies

\begin{tabular}{lll}
\hline \multicolumn{3}{c}{ Table 2: Relationships between ATPO and TSH } \\
\hline Entity & Value obtained & $p$-value \\
\hline Correlation ( r-value) & 0.45 & $<0.00001$ \\
Regression & 0.21 & \\
\hline
\end{tabular}

Table 3: Relationship between ATPO and Vitamin D

\begin{tabular}{lll}
\hline Entity & Value obtained & $p$-value \\
\hline Correlation(r-value) & -0.32 & $<0.00001$ \\
Regression & 0.10 & \\
\hline
\end{tabular}

Table 4: Relationship between ATPO and PTH

\begin{tabular}{lll}
\hline Entity & Value obtained & $p$-value \\
\hline Correlation (r-value) & 0.31 & \\
Regression & 0.094 & $<0.001$ \\
\hline
\end{tabular}

In this study, significant correlations $(\mathrm{p}<0.05)$ between ATPO and TSH (Table 2 and Fig. 4), vitamin D (Table 3, and Fig. 5) and PTH levels (Table 4) were found. Thyroid autoantibodies were inclined to be higher with lower vitamin D levels and higher TSH levels. A statistically significant negative correlation between vitamin $\mathrm{D}$ and PTH levels $(r=-0.27, p<0.004)$ Table 5 was found in the present study.

In our study, no statistical relation was found between with TSH, FT3, FT4 and vitamin D as their $p$-value is 0.066 , $0.53,0.83$ respectively and the entities have statistically significant relation only if their $p$-value is $\leq 0.05$.

\section{DISCUSSION}

Vitamin $D$ is a significant immunomodulatory as $1,25(\mathrm{OH})_{2} \mathrm{D}$ inhibits Th1 cell and B cell proliferation, differentiation into plasma cells, and production of cytokines (IL-2 and interferon- $\gamma$ ). The $1,25(\mathrm{OH})_{2} \mathrm{D}$ also enhances anti-inflammatory Th2 cytokines (IL-3, IL-4, IL-5, and IL-10) production and also induces $B$ cell apoptosis. The ability of $1,25(\mathrm{OH})_{2} \mathrm{D}$ to suppress the adaptive immune system promotes immune tolerance and appears to be beneficial for a number of autoimmune diseases like AITDS. ${ }^{78}$ Serum $25(\mathrm{OH})_{2}$ D estimation is the best pointer of vitamin D status. It reflects vitamin D delivered cutane-

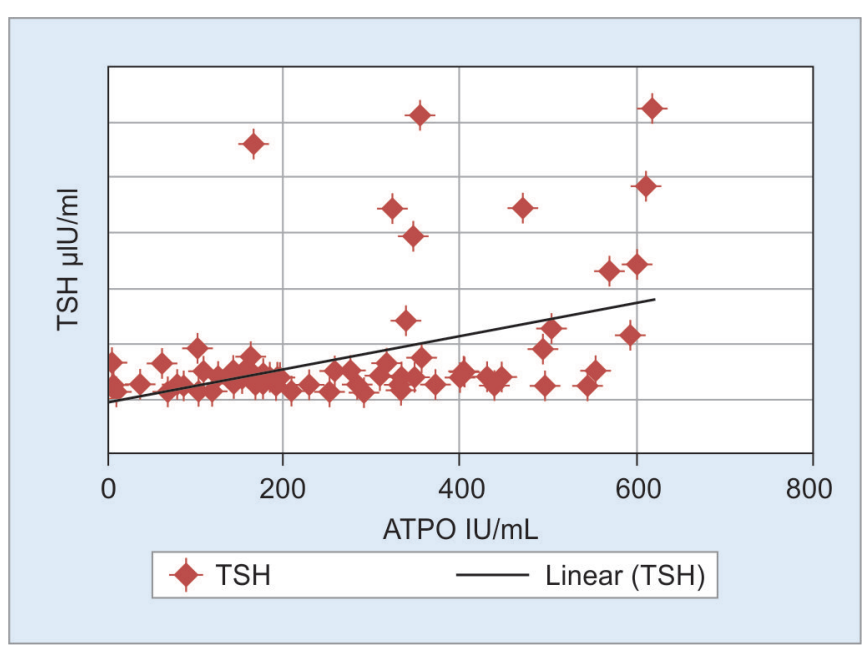

Fig. 4: Correlation between ATPO and TSH levels in hypothyroid patients
Table 5: Relationship between vitamin D and PTH

\begin{tabular}{lll}
\hline Entity & Value obtained & $p$-value \\
\hline Correlation (r-value) & -0.27 & $<0.0049$ \\
Regression & 0.074 & \\
\hline
\end{tabular}

ously, and that acquired from nourishment and supplements. $1,25(\mathrm{OH})_{2} \mathrm{D}$ is not a good marker of vitamin $\mathrm{D}$ status since it has a short half-life of 15 hours and serum concentration is directed by parathyroid hormone, calcium, and phosphate. ${ }^{9}$ Levels of $1,25(\mathrm{OH})_{2}$ D don't diminish until vitamin $\mathrm{D}$ inadequacy is severe. ${ }^{10}$ Therefore, in the present study, we measured serum $25(\mathrm{OH})_{2}$ D instead of $1,25(\mathrm{OH})_{2} \mathrm{D}$. In past also some studies were conducted to find any significant relationship between vitamin D levels and AITD and to determine the role of vitamin D deficiency in the pathogenesis of AITD.

We in this study evaluated the levels of Vitamin D, PTH and calcium among patients with AITDs compared to healthy controls without any symptom of thyroid diseases. In recent years, there were few studies which demonstrated a higher prevalence of Vitamin D deficiency in HT or GD1 $1{ }^{11-14}$ but to our best knowledge, this is among first studies that evaluated the association between thyroid autoantibodies and vitamin D levels.

In one of the studies done by Unal AD et al. found that hyperthyroid patients more frequently had vitamin D deficiency. ${ }^{15}$ Our study also showed lower serum 25 $(\mathrm{OH})$ vitamin D levels in both hypo and hyperthyroidism than those of controls, which was both more prevalent and severe in hyperthyroid patients. Since few of earlier studies showed that vitamin D deficiency is a prevalent health problem, we accepted a cutoff of vitamin D level as $20 \mathrm{ng} / \mathrm{mL} \cdot{ }^{16}$ According to this cutoff value, vitamin D deficiency was present in $72.7 \%$ of our study population.

As per few of previous studies severity of hyperthyroidism is not correlated with vitamin D deficiency, ${ }^{17}$ and antithyroid drugs have immunosuppressive effects. In addition, thyroid hormones relatively affect the renal activity of 1a-hydroxylase and plasma 1,25(OH)2D

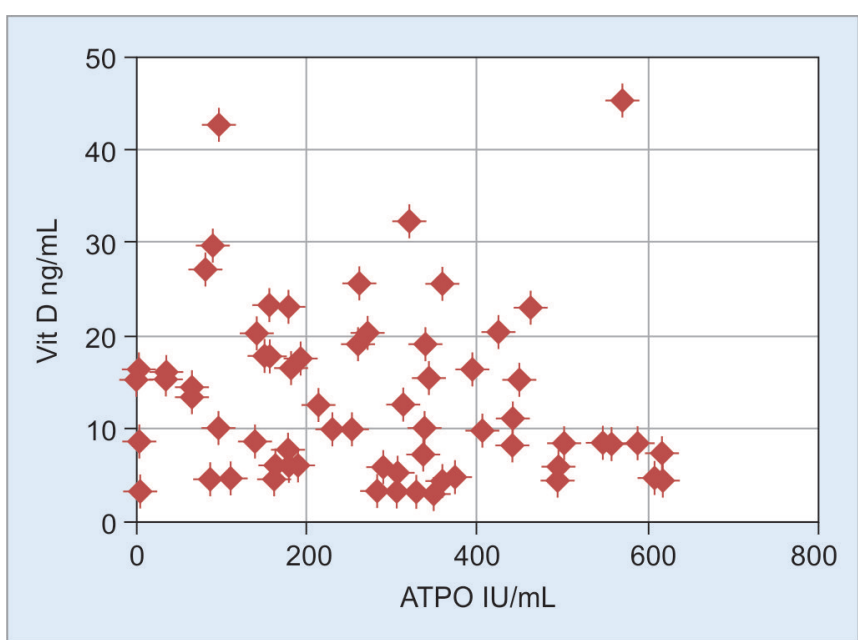

Fig 5: Correlation between ATPO and Vitamin D levels in Hypothyroid Group 
levels. ${ }^{18}$ For these reasons, only recently diagnosed AITDs patients were enrolled and we tested $25(\mathrm{OH}) \mathrm{D}$ level instead of $1,25(\mathrm{OH})_{2} \mathrm{D}$ in our study.

Our study found that in comparison to controls lower vitamin D and calcium serum levels were present in either group of ALTD patients (Table 1). But no significant correlation could be demonstrated between the patient's TSH levels and serum Vitamin D/Calcium levels.

Autoimmune diseases of the thyroid are comparatively common organ-specific autoimmune disorders which have variable presentations ranging from hyperthyroidism (GD) to hypothyroidism (HT). Loss of selftolerance, due to various genetic predispositions and environmental factors, may lead to the initiation of these AITDs. In chronic autoimmune conditions like thyroiditis, infiltrating lymphocytes, cell surface expression of MHCII, Fas-mediated apoptosis and cytokines released from both immune cells and thyrocytes contribute to amplification and progression of AITD. ${ }^{19,20}$ As per this theory, in the absence of functional suppressor T cells, Th-1 (CD4) cells can activate B lymphocytes to produce antibodies against multiple thyroid antigens $(\mathrm{TPO}, \mathrm{Tg}, \mathrm{TR}$, etc). Earlier studies on HT and GD patients had reported low vitamin D levels. ${ }^{13-15}$ Our study also reported that patients with autoimmune thyroiditis (GD group and HT group) present with lower vitamin D levels than controls. Also, the present study found a significant correlation between vitamin D, PTH, and ATPO levels. We recorded a significant negative association of Vitamin $\mathrm{D}$ with ATPO and PTH levels $(\mathrm{r}=-0.32, \mathrm{p}=0.0008: \mathrm{r}=$ $-0.27, p=0.0049)$ while significant positive correlation was found between PTH and ATPO levels ( $r=0.31, p=$ 0.001 ) among groups. Our results proposed that there may be a significant association between autoimmunity and vitamin D deficiency.

\section{CONCLUSION}

Our study demonstrated that lower vitamin D levels are found in patients with AITD at the time of diagnosis. Both higher prevalence and severity is seen in patients with GD. An inverse correlation between vitamin D levels and thyroid antibody levels was found in this study. As no intervention with Vitamin D supplements was tried in the present study, hence the above findings do not clarify whether treatment with vitamin D has any beneficial effect on the progression or remission of AITD. So, further studies specifically designed to evaluate the beneficial effect of vitamin D supplementation on AITD are needed.

\section{CLINICAL SIGNIFICANCE}

There is a need for large and well- designed prospective, adequately powered, community-based studies on prevalence, effect, and treatment efficacy in relation to the AITD keeping in mind of antithyroid peroxidase antibodies (ATPO-Abs) and Vitamin D relationship. This will enable a comprehensive and holistic approach to the treatment of AITDs.
Moreover, prospective studies with data regarding TSH, Calcium, and PTH will provide a basis for improving quality of care by health professionals.

\section{REFERENCES}

1. Dayan, Dayan, Colin M; Dayan, Colin M.; Gilbert H. Daniels (1996). "Chronic Autoimmune Thyroiditis". The New England Journal of Medicine. 335 (2):99-107.

2. Baeke F, Takiishi T, Korf H, et al. Vitamin D: modulator of the immune system. Curr Opin Pharmacol. 2010;10:482-496.

3. Cutolo M, Otsa K, Uprus M, et al. Vitamin D in rheumatoid arthritis. Autoimmun Rev. 2007;7:59-64.

4. Mok CC. Vitamin D and systemic lupus erythematosus: an update. Expert Rev Clin Immunol. 2013;9:453-463.

5. Iruretagoyena M, Hirigoyen D, Naves R, Burgos PI. Immune response modulation by vitamin $\mathrm{D}$ : role in systemic lupus erythematosus. Front Immunol. 2015;6:513.

6. Feng, M., Li, H., Chen, SF. et al. Polymorphisms in the vitamin D receptor gene and risk of autoimmune thyroid diseases: a meta-analysis. Endocrine 2013;43:318-326.

7. Kmie P, Sworczak K Vitamin D in thyroid disorders. Exp. Clin. Endocrinol. Diabetes. 2015;123:386-393

8. Prietl B, Treiber G, Pieber TR, Amrein K Vitamin D and immune function. Nutrients. 2013;5:2502-2521

9. Institute of Medicine, Food and Nutrition Board. Dietary Reference Intakes for Calcium and Vitamin D. Washington, DC: National Academy Press; 2010.

10. Elizabeth A, Brulé Danielle, Cindy D, Krista A, Peter WF, Friedl Karl E, et al. Dietary Reference Intakes for vitamin D: justification for a review of the 1997 values. Am J Clin Nutr. 2009;89(3):719-727.

11. Kim D. The Role of Vitamin D in Thyroid Diseases. International Journal of Molecular Sciences. 2017;18(9):1949.

12. Tamer G, Arik S, Tamer I, Coksert D. Relative vitamin D insufficiency in Hashimoto's thyroiditis. Thyroid. 2011;21: 891-896.

13. Bozkurt NC, Karbek B, Ucan B, et al. The association between severity of vitamin D deficiency and Hashimoto's thyroiditis. Endocr Pract. 2013;19:479-484.

14. Kivity S, Agmon-Levin N, Zisappl M, et al. Vitamin D and autoimmune thyroid diseases. Cell Mol Immunol. 2011;8:243247.

15. Unal AD, Tarcin O, Parildar H, Cigerli O, Eroglu H, Demirag NG. Vitamin D deficiency is related to thyroid antibodies in autoimmune thyroiditis. Cent Eur J Immunol. 2014;39(4):493-7.

16. Holick MF. Vitamin D deficiency. N Engl J Med 2007;357:266281.

17. Misharin A, Hewison M, Chen CR, et al. Vitamin D deficiency modulates Graves' hyperthyroidism induced in BALB/C mice by thyrotropin receptor immunization. Endocrinology. 2009;150:1051-1060.

18. Kozai M, Yamamoto H, Ishiguro M, et al. Thyroid hormones decrease plasma 1alpha,25-dihydroxyvitamin D levels through transcriptional repression of the renal 25-hydroxyvitaminD3 1alpha-hydroxylase gene (CYP27B1) Endocrinology. 2013;154:609-622.

19. Klecha AJ, Barreiro Arcos ML, Frick L, Genaro AM, Cremaschi G. Immune-endocrine interactions in autoimmune thyroid diseases. Neuroimmunomodulation. 2008;15:68-75

20. McLachlan SM, Nagayama Y, Rapoport B. Insight into Graves' hyperthyroidism from animal models. Endocr Rev. 2005;26:800-832. 